\title{
HE-ABNORMAL STARS
}

\section{KURT HUNGER and DETLEV GROOTE}

Institut für Theoretische Physik und Sternwarte, Universität Kiel, Olshausenstrasse 40, W-2300 Kiel, Federal Republic of Germany

\begin{abstract}
The He-rich variable HD 37479 has 2 axes of symmetry, one characterized by the depletion of metals, and one by the enrichment of $\mathrm{He}$. The former is oriented along the axis of the magnetic dipole, while the latter is off set by some $45^{\circ}$. The 2 axes represent 2 different modes of diffusion, the first one being controlled solely by the magnetic field, irrespective of wind, the second one being due to stellar wind that is controlled by the magnetic field and intertial forces. It has been attempted to formulate simple physical conditions that allow to determine the diffusion regions on the surface. It is shown that the resulting surface map can well reproduce the observed phase variations of the equivalent widths of $\mathrm{HeI} 4471$, of UV resonance lines of C IV and Si IV, and the effective magnetic field.
\end{abstract}

\section{INTRODUCTION}

This talk will be devoted mostly to the He-rich among the He-abnormal stars. In these stars, the number ratio of $\mathrm{He}$ to $\mathrm{H}$ is unity, typically. So far 24 stars brighter than $V=11^{\mathrm{m}}$ are known. Their spectral type is remarkably uniform, B2V, with $\mathrm{T}_{\text {eff }}$ ranging from $20000 \mathrm{~K}$ to $25000 \mathrm{~K}$. In many respects, they represent near main sequence objects (pre or post m.s.), with gravity slightly reduced, with "normal" masses and luminosities, and also "normal" distribution of rotational velocities. Outstanding, however, are the magnetic fields which are observed in 9 stars, and which are the largest observed in non-degenerates. A further anomaly is the array of variabilities as observed in 7 stars which comprises photometry, spectroscopy, magnetic field and polarization, and which have a common period (typically of the order of $1 \mathrm{~d}$ ), which points to oblique rotators. 
It also must be noted that a fraction exhibits circumstellar matter, by radio-emission, $\mathbf{H \alpha}$-emission and absorption, $\mathrm{X}$-rays and stellar wind. Also noteworthy is that a significant number of these stars are found in the Orion $\mathrm{OB} 1$ association.

The best studied object is HD 37479 which exhibits all of the above named features. The model is sketched in Fig. 1. It represents a star with 2 corotating clouds, with the axis of rotation vertical in the diagram, a nearly centered dipole, marked by + and -, 2 almost symmetric He-caps

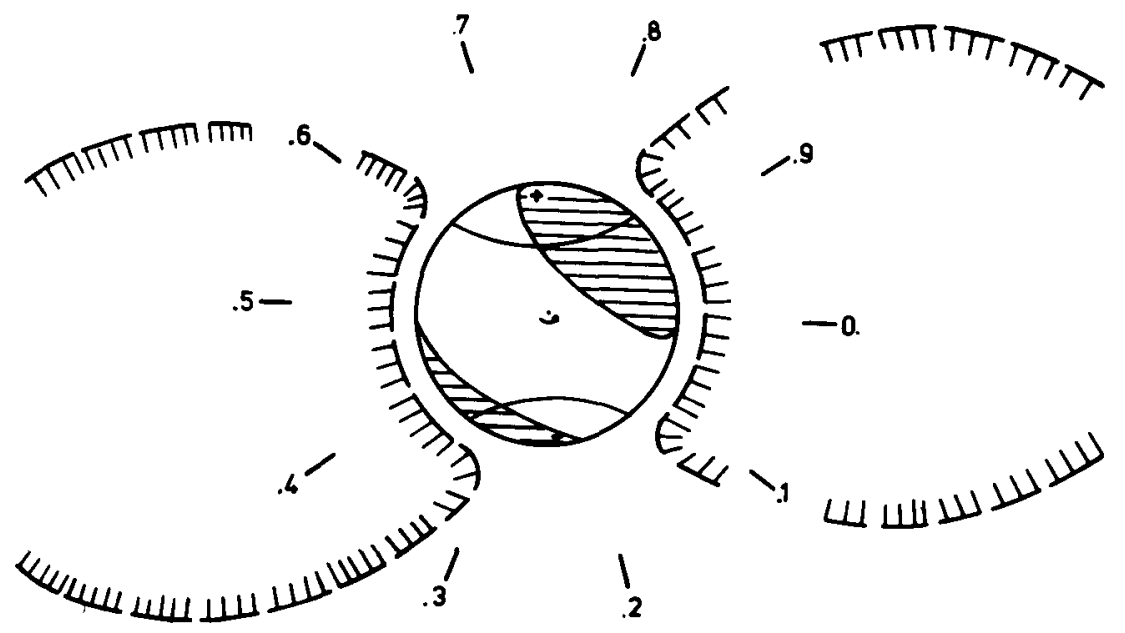

Figure 1: Model of HD 37479. He-caps are hatched. The magnetic poles are indicated by + and - . The circular section around the poles mark the regions of metal depletion. The clouds corotate. Phases are designated by their mumbers. 
(hatched) and 2 metal-depletion caps centered at the magnetic poles. Heenrichment and metal depletion is believed to be due diffusion. As the symmetry axes are off by some $45^{\circ}$, one is forced to assume that there are 2 different modes of diffusion operative:

one which is (solely) controlled by the magnetic field (besides gravity) and which leads to the depletion of metals

one which is controlled by the magnetic field and rotation as the He-caps are found near the intersection of the magnetic and rotational equators. (Bohlender 1988, Hunger et al. 1990).

\section{POLAR DIFFUSION}

The surface area in which the polar diffusion takes place is marked by the depletion of metals. The determination of the photospheric abundances is beset with the following problems: the optical lines are weak $\left(\mathrm{W}_{\lambda} \approx 0.2 \AA, \quad \mathrm{C}\right.$ II 4267$)$ with small phase variations $\left(\Delta W_{\lambda} / W_{\lambda} \approx 0.2\right)$, since the lines are on the flat part of the curve of growth. Moreover, Zeeman splitting may be important. The UV lines, on the other hand, are strong ( $W_{\lambda} \approx 1 \AA$, C IV 1548, Si IV 1393), with larger phase variation $\left(\Delta W_{\lambda} / W_{\lambda} \approx 0.3\right)$, as they are on the damping part of the c.o.g. However, the lines are (mostly) blended, the continuum is ill defined, NLTE-effects are large (of the order of a factor of 2 for $W_{\lambda}$ ), as the lines in question are resonance lines, and last not least, the lines are wind contaminated. In both spectral domains, the variation of atmospheric structure due to the variation of the He-content has also to be taken into account (roughly a factor of 1.5 in $\mathrm{W}_{\lambda}$ for the UV lines).

Let us begin with the wind contamination. In Fig. 2, the CIV doublet is shown for 2 extreme phases, in which the clouds are in quadrature (pole-on) and in conjunction (equator-on), resp. In both phases, the asymmetric circumstellar part is clearly discernible. It extends to $600 \mathrm{~km} / \mathrm{s}$, with $W_{\lambda}$ abs $\approx 0,3 \AA$. There is also a red emission at $+160 \mathrm{~km} / \mathrm{s}$, with $W_{\lambda} \approx-0.1 \AA$. The differences between the 2 phases is fully symmetric and centered at the laboratory wave lengths. The conclusion is that the asymmetric part is independent of phase, i.e. the circumstellar (wind) material is located outside the clouds (10-20 $\left.R_{*}\right)$. The variation of the symmetric part reflects the photospheric abundance variation. The same result is obtained for the doublet of Si IV. Since NLTE results are still pending, only the optical LTE-results can be quoted: outside the polar caps (equator-on), $\mathrm{C}$ as well as $\mathrm{Si}$ appear solar, while in the the phase "pole-on" the hemisphere-averaged abundances are reduced by a factor of 2.5 (C II) and 3 (Si III). Depending on the model assumed, the depletion of the metals inside the polar caps amounts to a factor of 6-10. 


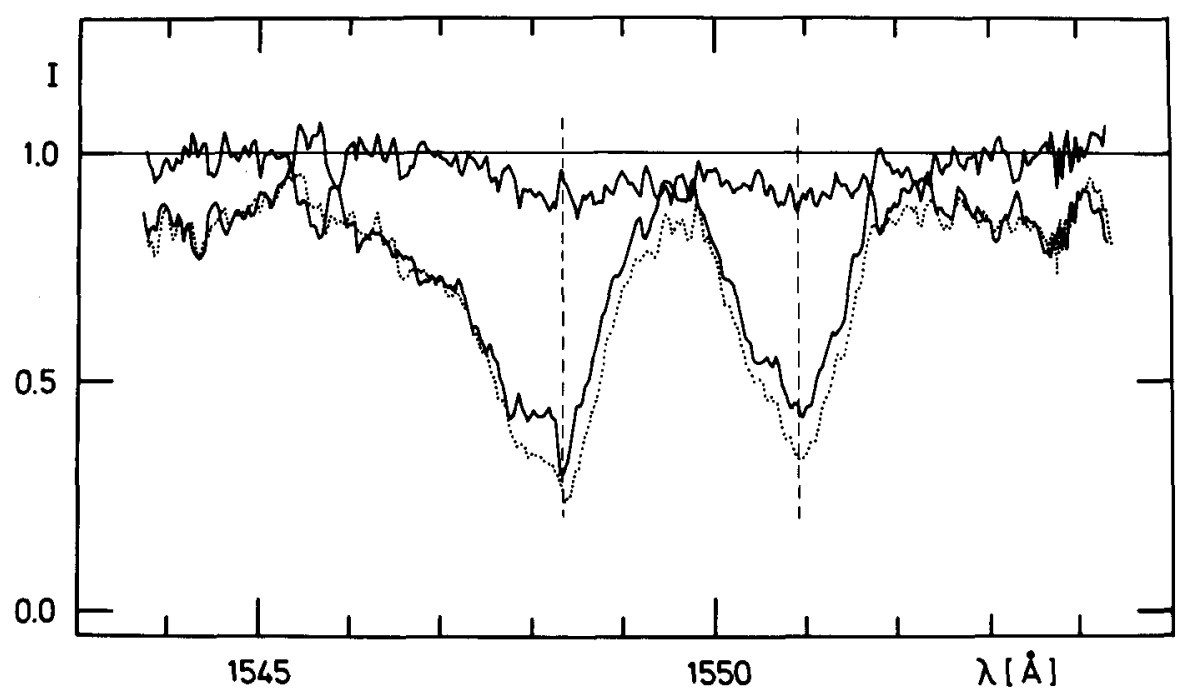

Figure 2: The C IV doublet at $\lambda 1548,1551$. Dotted: line profile at conjunction phase, thin lined at quadrature phase. (In order to reduce noise, 4 adjacent phases have been co added.) The difference of the 2 curves is shown at the top. Dashed lines mark the lab. wave lengths.

Irrespective of the exact abundances that may eventually be obtained, the following important statement can be expressed: the polar diffusion is well correlated with the magnetic field. $W_{\lambda} \max$ coincides with $\mathrm{B}_{\text {eff }}=0$, practically independent of $\mathrm{e} / \mathrm{m}$ (Hunger et al., 1990 and 1991). This is also true for HD 37017 and HD 64740 (both He-rich, Shore and Brown, 1990), and for HD 5737 and HD 79158 (Shore et al., 1990). As the latter two are He-weak stars, which are expected to have no wind or mass loss resp. in excess of $\mathrm{M}=10^{-13} \mathrm{M} \odot / \mathrm{yr}$, it means that the polar diffusion is controlled solely by the magnetic field irrespective of the presence of wind. For a "theoretical" surface mapping it is therefore sufficient to impose the condition $B>B_{0}$ (B is the local field, $B_{0}$ a free parameter) or, alternatively, $\alpha<\alpha_{0}$, where $\alpha$ is the zenith distance of the local field $B$, and $\alpha_{0}$ a free parameter. 


\section{MAGNETO-ROTATIONAL DIFFUSION}

The enrichment of $\mathrm{He}$ is believed to be caused by the wind, the exact mechanism yet unknown. The enrichment near the intersection of rotational and magnetic equators suggests that the wind is controlled by inertial forces (Bohlender, 1988) as well as Lorentz forces (Hunger et al. 1990). Centrifugal forces amount to less than $20 \%$ in gravity and hence can be neglected. Coriolis forces however are capable of preventing the onset of wind in certain parts of the surface ("angular momentum barrier") (Hunger et al. 1990).

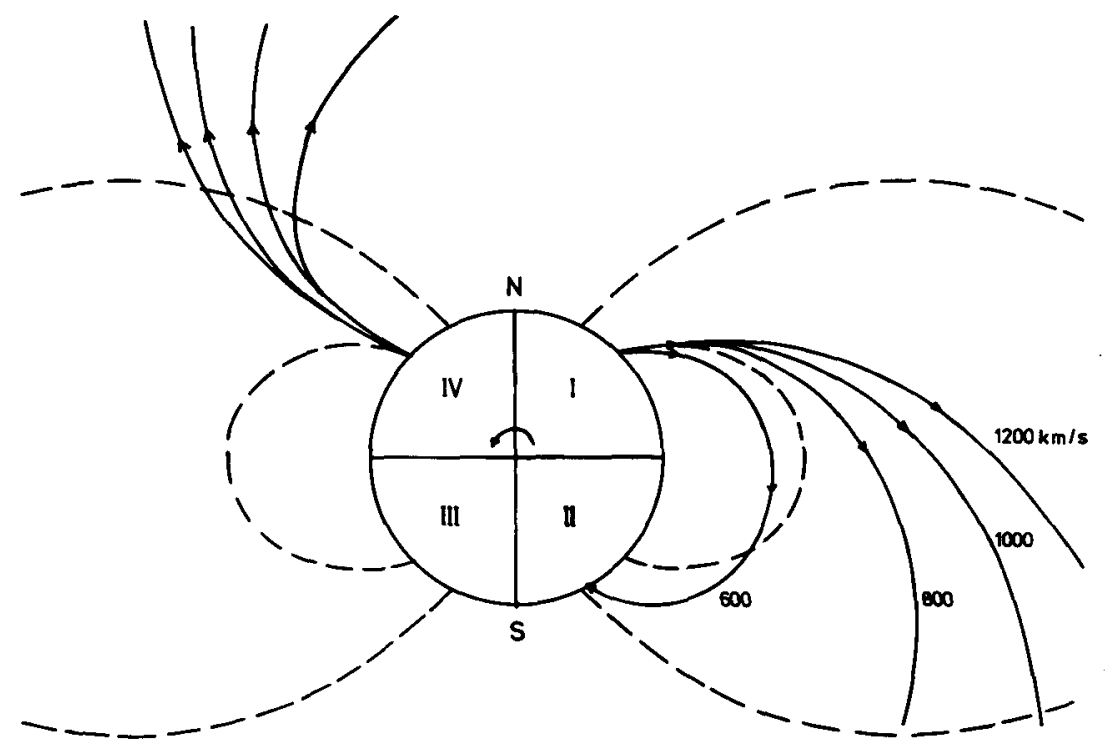

Figure 3: Approximate trajectories of wind particles that are subjected to gravity and inertial forces. Numbers quoted are initial velocities (see text!). 2 field lines are reproduced. Only in the first and third quadrant, field lines and trajectories have similar curvatures. 
The wind resp. mass loss has been observed in HD 37479: $M=10^{-10}-10^{-9} \mathrm{M} \odot / \mathrm{yr}$ (Hamann, 1981), in fair agreement with the (still provisional) theory: $M=1-410^{-9} \mathrm{M} \odot / \mathrm{yr}$ (Pauldrach, 1989). A large fraction (90\%) is injected into the clouds which extend up to a distance of $6 R_{*}$ (bound part of the wind). $10 \%$ freely escape from the polar caps (cap radius $\approx 24^{\circ}$ ). However, the angular momentum barrier prevents the wind, bound and free part, to emerge from the 2 . and 4 . quadrant (see Fig. 3).

To demonstrate the effect, we calculate the trajectories of the wind particles under the simplifying assumption that the particles are only subjected to gravity and Coriolis forces, and that the main radiative acceleration has occurred near the stellar surface, i.e. the particles have the quoted initial velocities. Moreover, the particles move in the (rotational) equatorial plane (fulldrawn). Next, the dipole field is added (field lines are dashed). Since the particles are forced to trail, only particles of the 1 . and 3 . quadrant will move more or less freely along the magnetic field lines. In the 2 . and 4 . quadrant, all trajectories cross the field lines, which means that all particles interact with the field and are eventually braked. So the wind is expected to arise predominantly from the 1 . and 3. quadrant, and these quadrants are (almost) identical with the He-rich surface regions.

The foregoing discussion is limited to the case of the equatorial plane. To define the wind regions outside this plane, some (approximate) necessary conditions can be formulated. Whether a particle can freely start from the surface, depends on the local field strength, on the local rotational velocity, and on the radiative acceleration which is peaked radially. Since the local field line is confined to a plane, the particle trajectory must also confined to a plane (preferentially to the plane of the field line). The latter, we call the local principle plane.

The equatorial plane (or its parallel) is another, invariant principle plane. A particle, initially moving in this plane, remains in this plane, inspite of Coriolis forces. A particle, with an initial velocity that has a transverse component will experience Coriolis accelerations that result in velocities inclined to the magnetic field. The condition that the wind particle moves in both principle planes, can only be met in the special case of the equatorial plane (and $B=90^{\circ}$ ) (see Fig. 3). For the general case (including the case $B \neq 90^{\circ}$, we have to allow some tolerance, i.e. that the two planes may intersect at small angles $\delta$ with $\delta<\delta_{0}\left(\delta_{0}\right.$ free parameter). $\delta$ is measured at the intersection of the 2 principle planes with the local tangential surface plane. 
The condition $\delta<\delta_{0}$ is largely determined by the Coriolis forces. A second condition is imposed by the Lorentz forces: the zenith distance of the local field must be small, $\alpha<\alpha_{0}$, otherwise the particle is forced to move in Larmor circles. $\alpha_{0}$ again, as in the polar diffusion case, is a free parameter. Its meaning, however, is different as in the polar case $\alpha_{0}$ stands for a sufficiently large $B_{0}$ with ensures that all motions are frozen and the atmosphere static, while in the magneto-rotational case $\alpha_{0}$ essentially determines the smallest acceptable Larmor radius. Hence in general, the $2 \alpha_{0}$ 's may take different values.

\section{SEMI-EMPIRICAL SURFACE MAPPING}

Given the magnetic field at the surface, for instance the field of a decentered dipole, plus the magnetic obliquity $B$ (i.e. the angle between the axis of rotation and the dipole), the 2 conditions

$$
\begin{aligned}
& \alpha<\alpha_{0} \\
& \delta<\delta_{0}
\end{aligned}
$$

determine the surface regions where the wind can emerge. (N.B. the above quoted conditions are necessary condition. There may be further conditions that restrict the wind regions. For instance the wind velocity must be such that the curvature of the wind trajectory roughly equals the curvature of the field line. In our example, the required velocities range from $250 \mathrm{~km} / \mathrm{s}$ at the equator to $800 \mathrm{~km} / \mathrm{s}$ at the pole. If the radiative acceleration is not sufficient, wind from the polar regions may be excluded.) Since the latter are assumed to be enriched in $\mathrm{He}$, the geometry of the He-patches can be determined.

For HD 37479 ample observational data with sufficient phase coverage exist, namly the equivalent widths $\mathrm{W}_{\lambda}$ of $\mathrm{He}$ I 4471 (Hunger et al. 1989, Groote and Hunger, 1977), the average $W_{\lambda}$ of C IV $(\lambda 1548,52)$ and Si IV $(1398,1403)$ and the effective magnetic field $B_{\text {eff }}$ (Bohlender, 1988) (see Fig. 4).

After some trials, the best fit to the observations was found with

$$
\begin{array}{ll}
\text { polar cap: } & \alpha_{0}=30^{\circ} \pm 5^{\circ} \\
\text { He-patches: } & \alpha_{0}=40^{\circ} \pm 10^{\circ} \\
& \delta_{0}=35^{\circ} \pm 5^{\circ}
\end{array}
$$

$\mathrm{W}_{\lambda}$ of C IV and Si IV, due to the lack of adequate NLTE line formation analyses (and the known problems with the continuum) are scaled to match the observations in the maximum phase. $\mathrm{W}_{\lambda}$ inside the polar caps are then assumed to be $1 / 5$ of $W_{\lambda}$ at maximum phase. (This ratio is obtained under LTE conditions. The results, however, do not depend critically on the precise ratio.) 


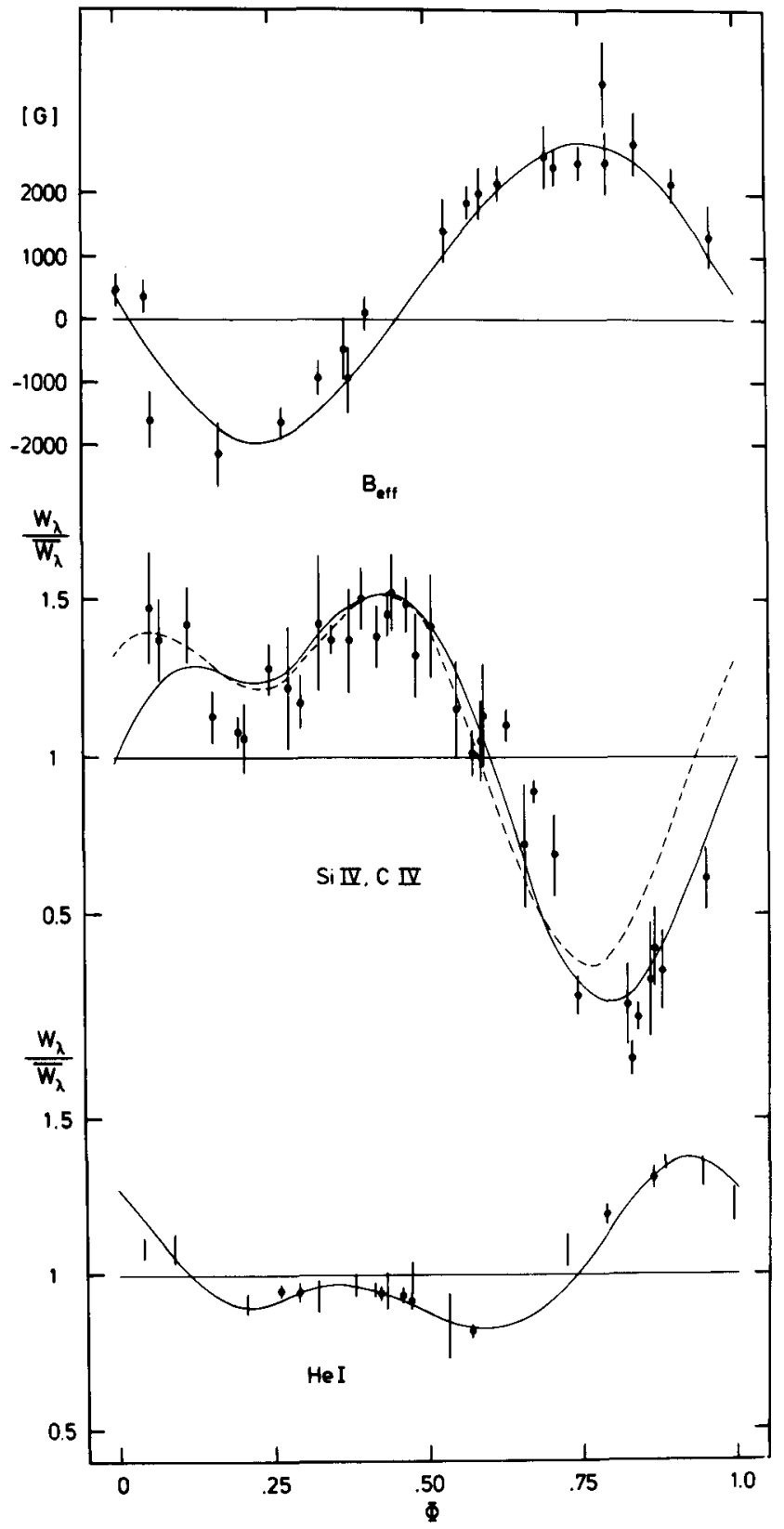

Figure 4: Phase dependance of $B_{\text {eff }}$ of $W_{\lambda}$ of Si IV and C IV, and $W_{\lambda}$ of He I 4471. Fulldrawn: semi-empirical model. (For the dashed curve, see text.) 
Inside the He-patches, $\epsilon_{\mathrm{He}}=0.90\left(\mathrm{~W}_{\lambda}=2.00 \pm 0.1\right)$ is assumed, while outside $\epsilon_{\mathrm{He}}=0.16\left(\mathrm{~W}_{\lambda}=0.77 \pm 0.02\right)$. This means, that even outside the patches, He appears enriched, or conversely, that some wind also occurs outside the patches. (A further test with $\epsilon_{\mathrm{He}}$ inside $=0.80\left(\mathrm{~W}_{\lambda}=1,86 \pm 0.1\right.$ ) has been made. It yields almost the same equivalent widths, though the surface map - see below - is somewhat different.)

The axis of the dipole is inclined with respect to the axis of rotaton: $B=73^{\circ} \pm 2^{\circ}$. The dipole is decentered: It is shifted to the +pole by $(0.08 \pm 0.02) R_{*}$ and is offset by $(0.09 \pm 0.03) R_{*}$ in the orthogonal direction. $B_{p}=10000 \pm 300 \mathrm{G}$ are assumed.

The inclination angle $i$ is mainly determined from the rotational period $P$ and the "spectroscopic" radius $R_{*}: i=56^{\circ} \pm 2^{\circ}$.

The theoretical phase variation is shown in Fig. 4 (full drawn curve). The agreement with the observations is remarkably good. the dashed curve for $W_{\lambda} C I V, S i$ IV is obtained when one neglects the effect that $\mathrm{W}_{\lambda}$ is reduced in the He-rich phase.

The "semi-empirical" surface map is shown for 4 phases in Fig. 5. He appears enriched in more or less triganular patches (vertical hatches), instead of the hitherto assumed circular caps. (In addition, the He-patch is shown for the case that the He-content inside the patch is $\epsilon_{\mathrm{He}}=0.80$, instead of 0.90 (dashed). Fig. 4, however, remains unchanged.)
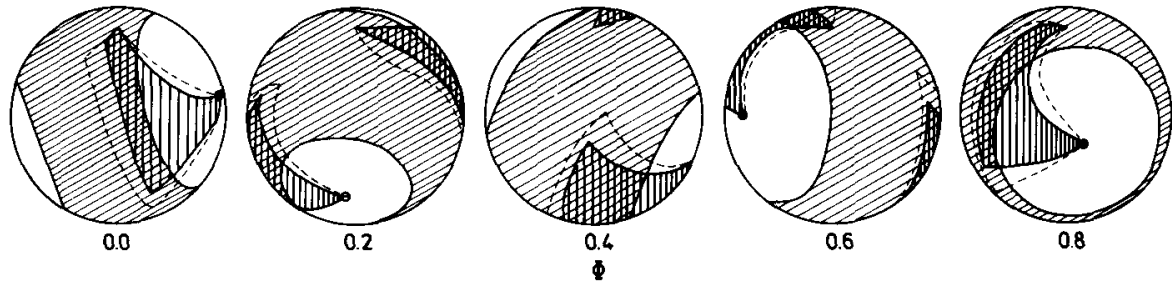

Figure 5; Semi-empirical surface map. Vertical hatches: He-patches; unhatched: metal poor caps. The dashed contour of the He-patches results when a slightly smaller Heabundance is assumed. 


\section{DISCUSSION}

The He-rich variable HD 37479 clearly exhibits 2 axes of symmetry, one marked by the depletion of metals and the other by the enrichment of helium. The former is oriented along the dipole axis, the latter is offset from this axis by roughly $45^{\circ}$.

While the polar (downward) diffusion of metals can be understood as being due to the magnetic field that stabilizes the atmosphere, the situation is less clear in the case of He-enrichment. The mere fact that He is enriched, instead of depleted as one would expect on account of its mass, points to the action of stellar wind -and the latter indeed is observed. The orientation of the symmetry axis can only be explained if one assumes that the wind ist controlled as well by magnetic as by inertial forces, of the latter the Coriolis forces being the more important. Due to the action of Coriolis forces (plus magnetic field), some surface areas will shed less wind than others, because, due to the "angular momentum barrier," additional acceleration is needed to lift a wind particle to its final orbit in the clouds (90\% of the wind is interim stored in the corotating clouds).

In order to define the wind region on the surface of a rotating and magnetized star, the 3-dimensional hydrodynamical problem of the radiation driven, magnetically controlled wind in a rotating system has to be solved. This is certainly beyond the scope of the present study. Instead simple approximate prescriptions are tentatively proposed, that allow to single out the regions where the wind is allowed. The first condition is that the motion of the radiation driven wind particle (predominantly radial) does not deviate too much from the local field (i.e. the zenith distance $\alpha$ of the local field vector be small), otherwise Lorentz forces become large. The second condition is that Coriolis forces have only small components which accelerate the wind particle away from the local field line which in turn would induce Lorentz forces. This condition is equivalent to the condition that the projection of the local field vector on the surface, has approximately the same direction as the local rotational velocity. The second condition is a necessary condition that can be expressed by $\delta<\delta_{0}$, where $\delta$ is the angle between the two vectors.

The two conditions for the He-patches and the one condition for the metal caps have been employed to derive a "semi-empirical" surface map, that leads to a satisfactory agreement between observations and theory. The method to arrive at a final model was that of trial and error. The degree of freedom one has is astronishingly small which is related to the fact that there is a close coupling between the magnetic intensity and the strength of the metal lines (the latter indeed can be used to supplement the magnetogram of the star). For the same reason, the uncertainties of the derived model parameters are also quite small, as has been shown in Sect. 4. 
The above described procedure has proved its usefulness in the one case presented. It would be of great importance to test further cases, those of He-rich and He-poor stars. The latter are especially interesting as they are known to have no wind. Diffusion in these stars should be limited to the polar regions.

\section{REFERENCES}

Bohlender, D.A., 1988, Thesis, University of Western Ontario, London, Ontario

Groote, D., Hunger, K., 1977, A\&A 56, 129

Groote, D., Hunger, K., 1982, A\&A 116, 64

Hamann, W.-R., 1981, private communication

Hunger, K., Heber, U., Groote, D., 1989, A\&A 224, 57

Hunger, K., Heber, U., Groote, D., 1990, in: Properties of Hot Luminous

Stars, Boulder-Munich Workshop, ed. C.D. Garmany, p. 307

Hunger, K., Groote, D., Heber, U., 1991, in: Evolution of Stars:

The Photospheric Abundance Connection, G. Michaud and

A. Tutukov, eds. IAU, p. 173

Pauldrach, A., 1989, private communication

Shore, S.N. and Brown, D.N., 1990, ApJ 365, 665

Shore, S.N., Brown, D.N., Sonneborn, G., Landstreet, J.D., Bohlender, D.A., 1990, ApJ 348, 242 